\section{Structural character of sorghum endosperm transfer cells and their relationship with embryo and endosperm}

\author{
Yankun Zheng, Zhong Wang \\ The Key Laboratory of Crop Physiology \\ and Genetics of Yangzhou University, \\ Yangzhou, Jiangsu Province, China
}

\begin{abstract}
Endosperm transfer cells mainly occur in the epithelial layer of the endosperm and transport the nutrient unloaded by the maternal vascular tissue. They have wall ingrowths that can facilitate solute transportation. Here we report our further investigation of endosperm transfer cells in sorghum (Sorghum bicolor L. Moench). We observed endosperm transfer cells, embryo, and endosperm with different kinds of microscopes. Our experimental results showed that the distribution and configuration of endosperm transfer cells were fit for solute transportation, and they had a tight relationship with the embryo and endosperm.
\end{abstract}

\section{Introduction}

Transfer cells (TC) are a special kind of cell with wall ingrowths. The plasma membrane grows clinging to the wall ingrowths and its superficial area increases greatly. Therefore, the surface-to-volume ratio of the protoplast is magnified, fit for absorbing and secreting substances. The wall ingrowths form as intensive transport starts and become best developed on those faces of cells presumed to be most active in solute transport. ${ }^{1}$

TC have been found in many grain caryopses. $^{2-6}$ In maize, the endosperm epithelial cells facing the placentochalazal area differentiate into endosperm transfer cells (ETC). ${ }^{7}$ The ETC are the only substance exchange zone between maternal and filial tissues. ${ }^{8}$ Davis and his coworkers investigated the ETC morphology in detail under the electron microscope. In cross-section, cell wall ingrowths appear as densely packed lobes, near which are mitochondria and endoplasmic reticulum.

In this report, we focus on structural characters of sorghum (Sorghum bicolor L. Moench) endosperm transfer cells and their relationship with the embryo and endosperm. The results enrich the knowledge of plant physiology and provide the theoretical basis for an increased yield of this crop.

\section{Materials and Methods}

\section{Plant material}

Sorghum plants (Sorghum bicolor L. Moench) grew in the experimental field of Yangzhou University, from July to September.

\section{Observation of endosperm transfer cells under a fluorescence \\ microscope}

Sorghum caryopses of different development stages were collected and then cut longitudinally into thin pieces with a refrigerated microtome (Leica CM1100). Then the ETC were observed under a fluorescence microscope (Leica DMLB).

\section{Observation of endosperm and embryo under a stereomicroscope}

The longitudinal sections of sorghum caryopses were stained with iodine-potassium iodide and triphenyltetrazolium chloride. Then both endosperm and embryo were observed under the stereomicroscope (Leica MZ6).

\section{Observation of endosperm transfer cells under light and transmission} electron microscopes

The observed region was cut into pieces of suitable size and fixed in $3 \%$ glutaraldehyde for $3 \mathrm{~h}$ or more. The samples were rinsed with $0.1 \mathrm{M}$ phosphate buffer $(\mathrm{pH} 7.4)$ three times and fixed again with $0.5 \% \mathrm{OsO}_{4}$ for $3 \mathrm{~h}$. Then, samples were rinsed with $0.1 \mathrm{M}$ phosphate buffer ( $\mathrm{pH}$ 7.4) three times and dehydrated in an ethanol series of $20 \%, 40 \%, 60 \%, 80 \%, 90 \%$, 95\% (15 min each), and 100\% (three times, 30 min each). Following this, ethanol was replaced by propylene oxide, and low glutinosity Spurr's resin was used to infiltrate and embed materials. Once dried at $70^{\circ} \mathrm{C}$ for $12 \mathrm{~h}$ in a thermostat, the samples were cut into $1000 \mathrm{~nm}$ thin sections, stained with toluidine blue, and observed under the light microscope (Leica DMLS). Based on this, samples were cut into $60 \mathrm{~nm}$ thin sections, stained with uranyl acetate and lead citrate, and observed under a transmission electron microscope (Philips Tecnai 12).

\section{Observation of endosperm transfer cells under the scanning electron microscope}

The observed region of sorghum caryopses was cut into pieces of suitable size under low temperature and fixed in $3 \%$ glutaraldehyde for at least $3 \mathrm{~h}$. After rinsing three times with $0.1 \mathrm{~mol} / \mathrm{mL}$ phosphate buffer ( $\mathrm{pH} 7.4$ ), samples were dehydrated in an ethanol series of $20 \%$, $40 \%, 60 \%, 80 \%, 90 \%, 95 \%$ (15 min each), and
Correspondence: Zhong Wang, The Key Laboratory of Crop Physiology and Genetics of Yangzhou University, Yangzhou, Jiangsu Province, China. E-mail: wangzhong@yzu.edu.cn

Key words: sorghum, endosperm transfer cells, wall ingrowths, endosperm, embryo.

Received for publication: 16 December 2009. Revision received: 5 August 2010.

Accepted for publication: 6 August 2010.

This work is licensed under a Creative Commons Attribution 3.0 License (by-nc 3.0).

CCopyright Y. Zheng, Z. Wang, 2010

Licensee PAGEPress, Italy

International Journal of Plant Biology 2010; 1:e15 doi:10.4081/pb.2010.e15

$100 \%$ (30 min). Then, ethanol was replaced by isoamyl acetate, and samples were dried by the supercritical fluid drying of $\mathrm{CO}_{2}$. After mounting on aluminum stubs with conducting carbon paint and sputter-coated with gold, samples were observed under a scanning electron microscope (Philips XL-30 ESEM).

\section{Results}

Distribution and structural character of endosperm transfer cells

The endosperm epithelial cells consisted of aleurone cells and ETC. ${ }^{9}$ At 6 days after pollin ation (DAP), radially the ETC lie near the maternal tissues, which comprise nucellar cells, placentochalazal cells, and vascular bundles from the inner to the outer layers (Figure 1B). Periclinally the ETC are adjacent to the aleurone cells (Figure 1B). There is one layer of ETC with a few wall ingrowths (Figure 1B). At 9 DAP, nucellar cells adjacent to the ETC disappear and the endosperm cavity forms (Figure 1C). The wall ingrowths of the ETC increase (Figure 1C). At 15 DAP, the zone around the ETC shows a dark color (Figure 1A). At 20 DAP, the endosperm cavity is very obvious (Figure 1D and E). One side distributes the ETC and the other lays dense vascular bundles (Figure 1E). Wall ingrowths are very thick (Figure 1D) and like a flange (Figure 1F).

When cells are cut transversely, wall ingrowths of the ETC appear to grow around the wall (Figure 2A). There are vacuoles of different sizes in the ETC (Figure 2B and C). There are also some uncertain organelles with surrounding thick garland-like structures in the ETC (Figure 2B). Many mitochondria (Figure 2E) and a dense endoplasmic reticulum (Figure 2F) are closely related to the wall ingrowths. The part of the nucleus opposite the wall ingrowths 

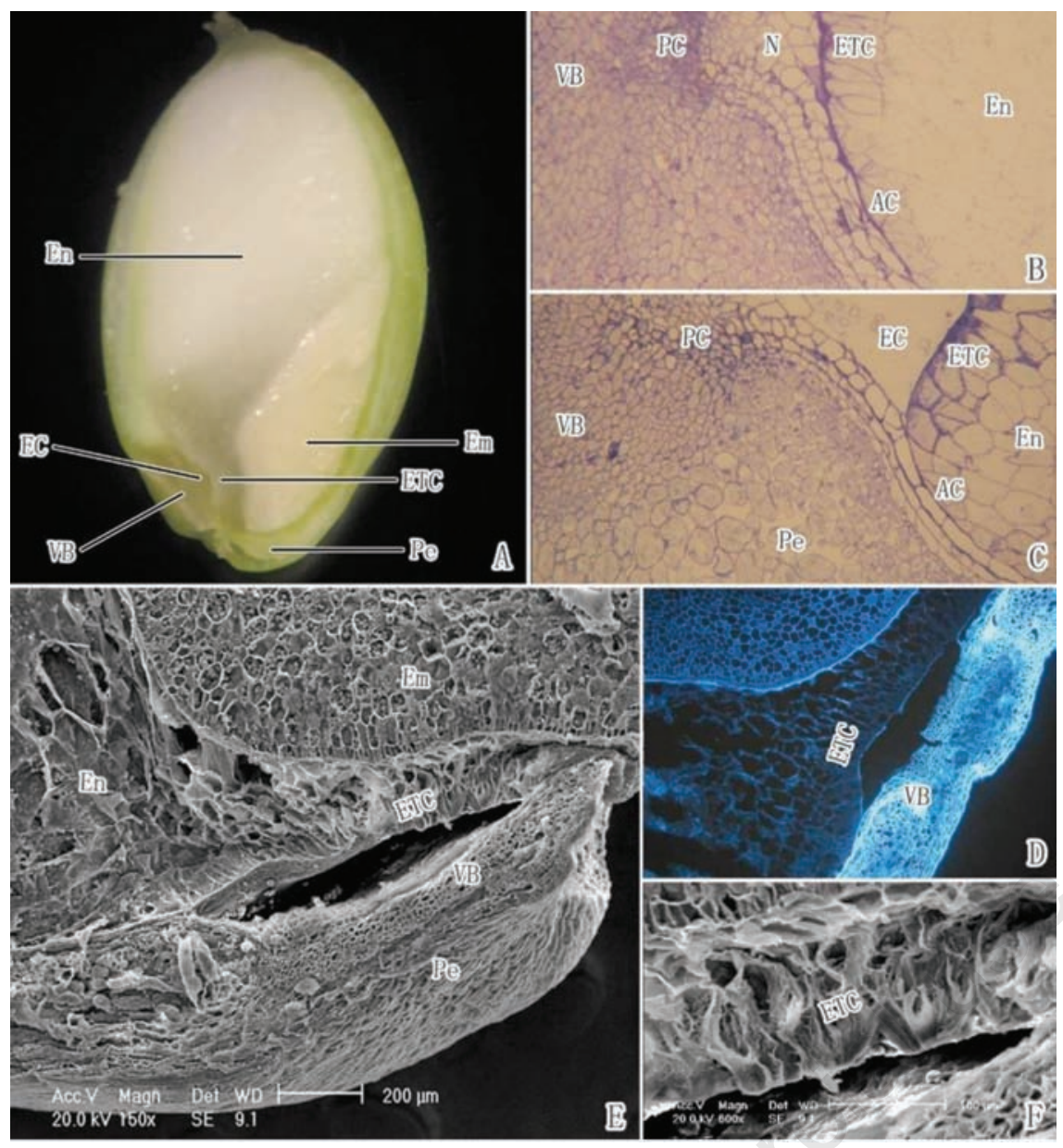

Figure 1. The distribution and configuration of sorghum endosperm transfer cells. (A) longitudinal section of the sorghum caryopsis under the stereomicroscope (magnification: $15 \mathrm{X})$; $(\mathrm{B}, \mathrm{C})$ region between the filial and maternal tissues under the light microscope (200X); (D) sorghum endosperm transfer cells under the fluorescence microscope $(100 \mathrm{X})$; (E,F) sorghum endosperm transfer cells under the scanning electron microscope (E: 150X, F: 600X). A, 15 DAP; B, 6 DAP; C, 9 DAP; D,E,F, 20 DAP; En, endosperm; Em, embyo; ETC, endosperm transfer cells; EC, endosperm cavity; VB, vascular bundles; Pe, pericarp; PC, placentochalazal cells; AC, aleurone cells; N, nucellar cells.
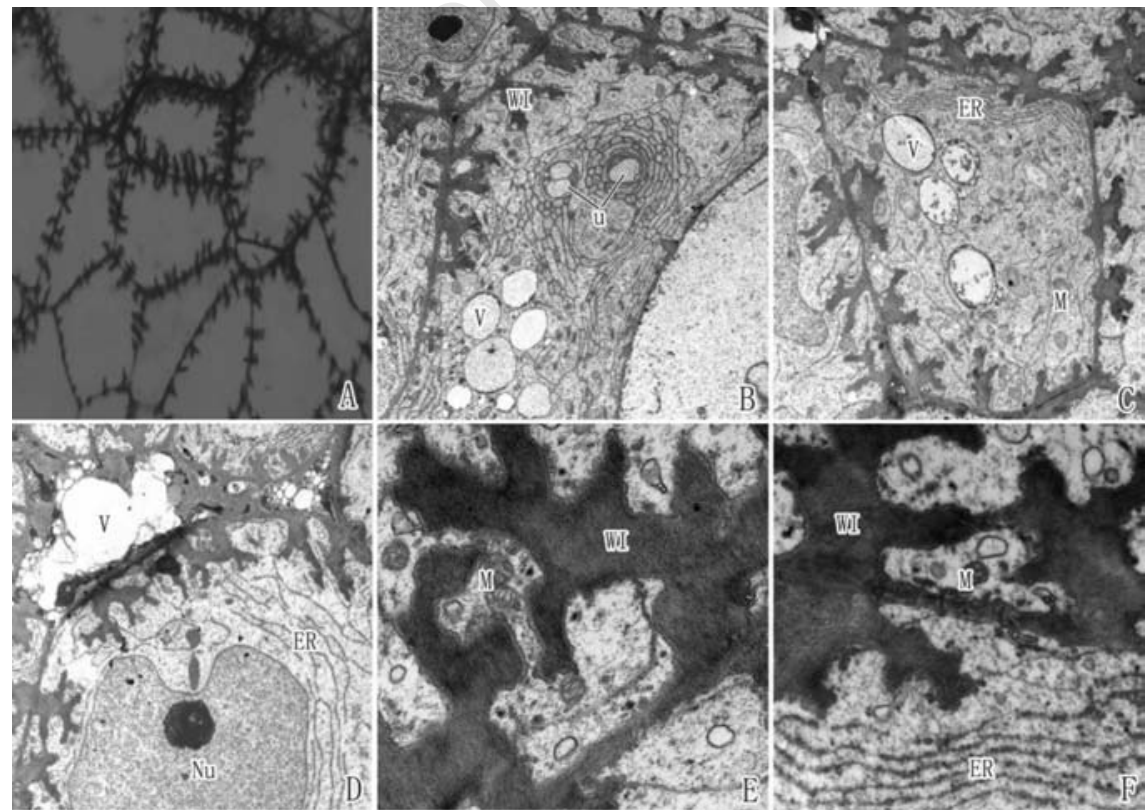

invaginates and the endoplasmic reticulum near the nucleus is distributed in the direction of invagination (Figure 2D).

When cells are cut longitudinally, wall ingrowths of the ETC are of polarity. Only the side and bottom walls near the maternal tissues form wall ingrowths (Figure 3B-H). Wall ingrowths grow less and less from the outermost to the inner layers and form firstly in the outermost layer and later in the inner layer (Figure 3A-H).

The relationship of the endosperm transfer cells with the embryo and endosperm

At 6 DAP, there are a few wall ingrowths connected to the bottom wall of the first-layer cells (Figure 3A). At 9 DAP, wall ingrowths of the first-layer cells increase slightly, and a part of their side wall and some second-layer cells have wall ingrowths (Figure 3B). At 12 DAP, wall ingrowths of both the first-layer and second-layer cells increase slightly (Figure $3 \mathrm{C}$ ). At $15 \mathrm{DAP}$, wall ingrowths of the first-layer cells increase markedly (Figure 3D). At 18 DAP, wall ingrowths of the first-layer cells continue increasing and become very thick (Figure $3 \mathrm{E}$ ). At 21 DAP, wall ingrowths are almost the same as at 18 DAP (Figure 3F). At 24 DAP, wall ingrowths crimp and decrease, but not obviously (Figure 3G). At 27 DAP, wall ingrowths crimp and decrease continually, and those of some second-layer cells almost disappear (Figure $3 \mathrm{H}$ and I). In summary, at about $6 \mathrm{DAP}$, wall ingrowths of the ETC begin forming; between 6 and 12 DAP, wall ingrowths of the ETC increase slowly; between 12 and 18 DAP, wall ingrowths of the ETC increase rapidly; between 18 and $24 \mathrm{DAP}$, wall ingrowths of the ETC keep almost the same dense configur-
Figure 2. Structure of sorghum endosperm transfer cells (cells cut transversely). (A) sorghum endosperm transfer cells under the light microscope (magnification: $1000 \mathrm{X}) ;(\mathrm{B}, \mathrm{C}, \mathrm{D})$ sorghum endosperm transfer cells under the transmission electron microscope (3900X); (E,F) wall ingrowths of sorghum endosperm transfer cells under the transmission electron microscope (18500X). WI, wall ingrowths; $M$, mitochondrion; ER, endoplasmic reticulum; V, vacuole; $\mathrm{Nu}$, nucleus; $\mathrm{u}$, uncertain organelle. 
ation; between 24 and 27 DAP, wall ingrowths of the ETC crimp and decrease.

Between 3 and 6 DAP, there is only a little starch accumulated in the endosperm (Figure $4 \mathrm{~A}$ and $\mathrm{B}$ ) and the embryo is not obvious (Figure 5A and B). Between 6 and 12 DAP, the starch of the endosperm is accumulated slowly (Figure 4B-D) and the embryo grows slowly (Figure 5B-D). Between 12 and 18 DAP, the starch of the endosperm is accumulated more rapidly (Figure 4D-F) and the embryo grows more quickly (Figure 5D-F). Between 18 and 24 DAP, there is a large starch accumulation in the endosperm (Figure 4F-H) and a significant growth of the embryo (Figure 5F-H). Between 24 and 27 DAP, both endosperm (Figure $4 \mathrm{H}$ and I) and embryo (Figure 5H and I) do not change obviously.

The ETC with wall ingrowths are the only substance exchange zone between maternal and filial tissues, ${ }^{8}$ and wall ingrowths facilitate nutrient transport. ${ }^{70}$ At about 6 DAP, wall ingrowths of the ETC begin forming and the ability of the ETC for substance transport is weak. Thus, there is very little nutrient transported to the filial tissues before 6 DAP. This is probably the main reason why the endosperm and embryo are not obvious between 3 and 6 DAP. Likewise, between 6 and 12 DAP, the slow development of the endosperm and embryo is probably correlated to the slow growth of the ETC wall ingrowths. From 12 to 18 DAP, the more rapid development of the endosperm and embryo is probably ascribed to the fast growth of the ETC wall ingrowths, while between 18 and 24 DAP, the obvious changes of the endosperm and embryo are likely related to the persistent dense configuration of the ETC wall ingrowths. From 24 to 27 DAP, the non-obvious changes of the endosperm and embryo are probably because of the crimping and decreasing of the ETC wall ingrowths. In one word, the ETC have a tight relationship with the embryo and endosperm.

\section{Discussion}

The developing seeds are strong sinks with no symplastic connection with the mother plant, as they are distinct individuals. Consequently, during the grain filling phase seed storage organs must develop specialized structures to facilitate nutrient uptake from the apoplastic space..$^{11}$ According to our results, we know that: in sorghum, the endosperm epithelial cells radially adjacent to the maternal tissues (from inner to outer: nucellar cells, placentochalazal cells, and vascular bundles) differentiate into ETC at about 6 DAP. The other epithelial cells differentiate into aleur one cells. After 9 DAP, nucellar cells adjacent to the ETC decrease and the endosperm cavity

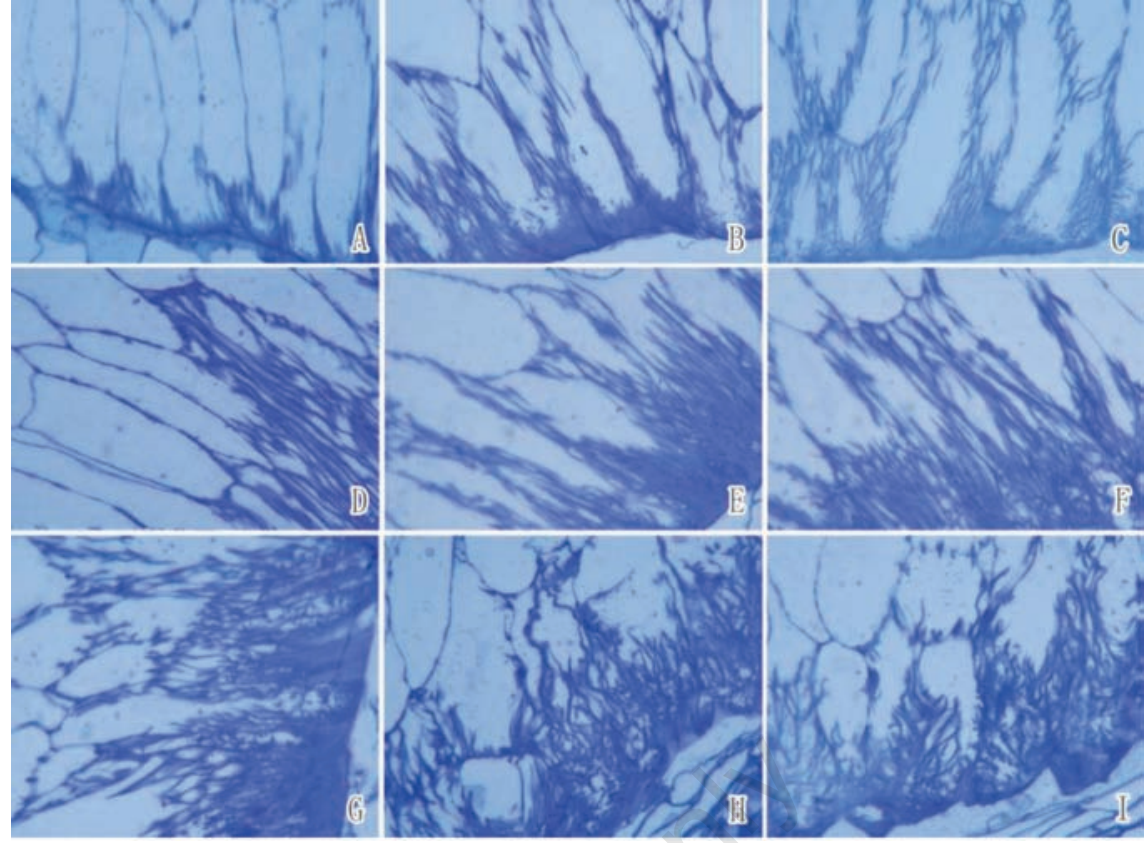

Figure 3. Sorghum endosperm transfer cells at different days after pollination (DAP) under the light microscope (magnification: 1000X, cells cut longitudinally). A, 6 DAP; B, 9 DAP; C, 12 DAP; D, 15 DAP; E, 18 DAP; F, 21 DAP; G, 24 DAP; H,I, 27 DAP.
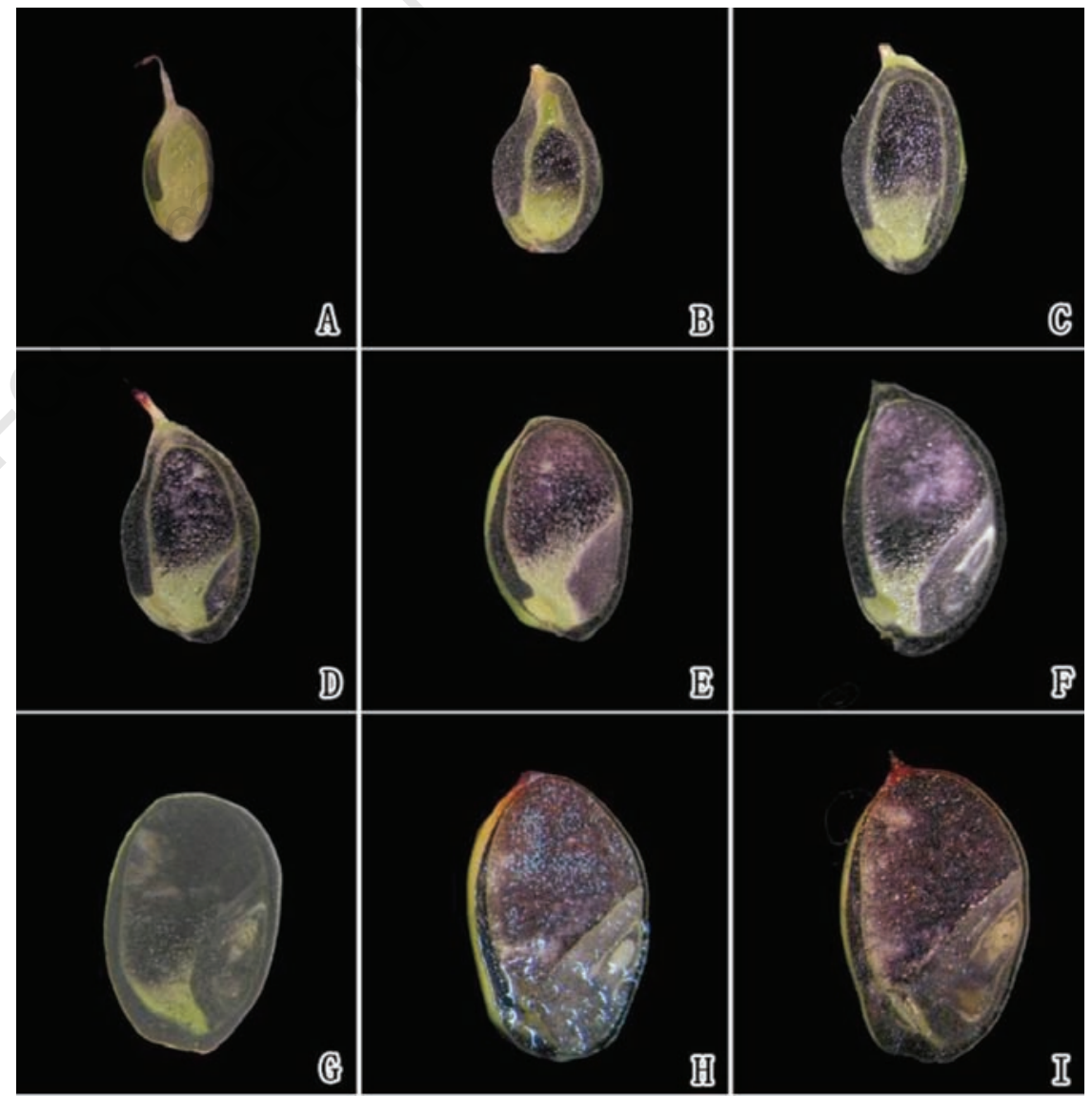

Figure 4. Sorghum endosperm at different days after pollination (DAP) under the stereo microscope. A, 3 DAP; B, 6 DAP; C, 9 DAP; D, 12 DAP; E, 15 DAP; F, 18 DAP; G, 21 DAP; H, 24 DAP; I, 27 DAP. 

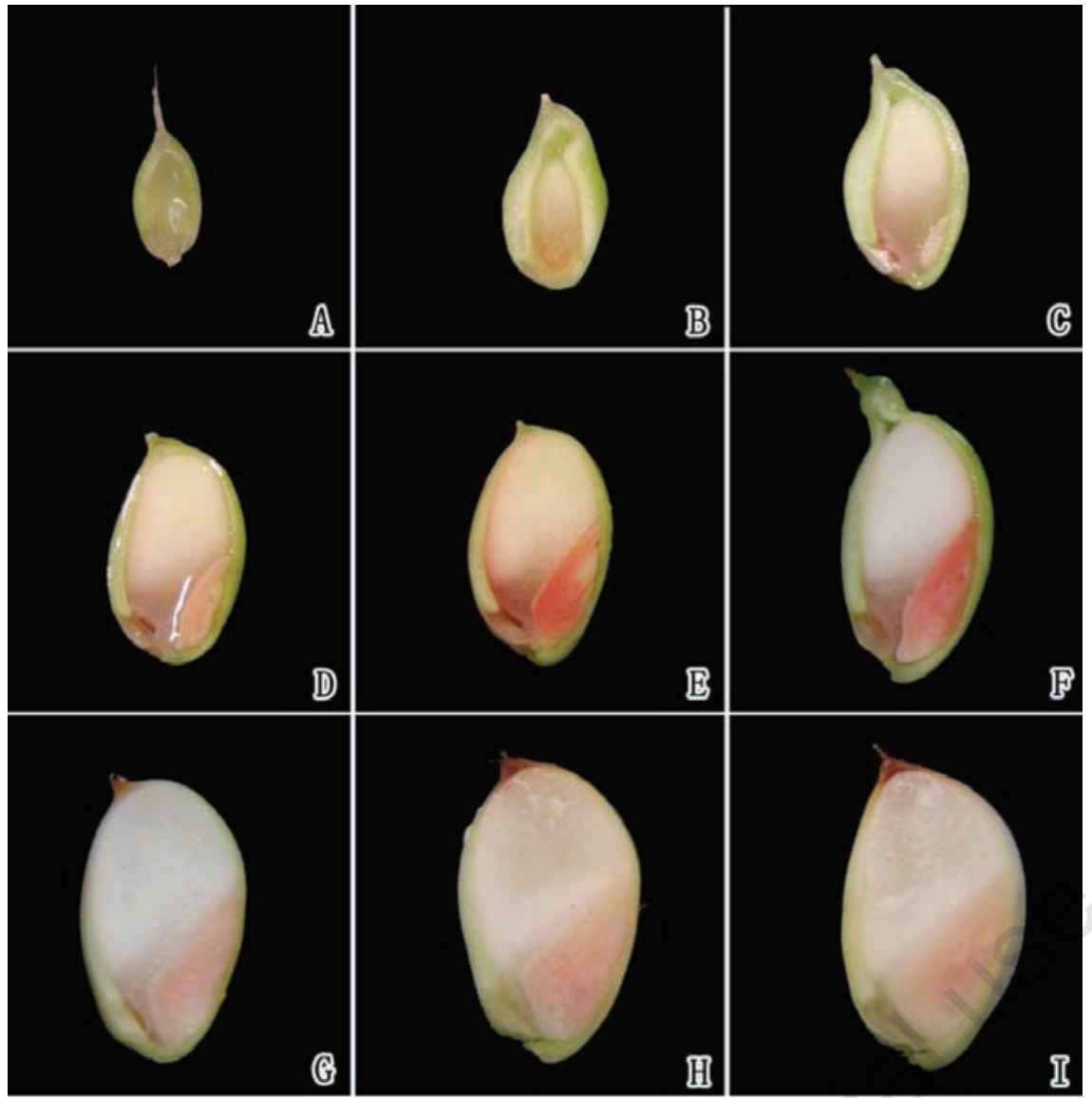

Figure 5. Sorghum embryo at different days after pollination (DAP) under the stereomicroscope. A, 3 DAP; B, 6 DAP; C, 9 DAP; D, 12 DAP; E, 15 DAP; F, 18 DAP; G, 21 DAP; H, 24 DAP; I, 27 DAP.

forms step by step. The nutrient unloaded by the vascular terminals traverses the placentochalazal cells and into the endosperm cavity. ${ }^{12}$ Finally, the nutrient is transported into the filial tissues by the ETC. ${ }^{8}$

The wall ingrowths of transfer cells can increase the substance-exchanging area of the plasma membrane and promote nutrient transport. ${ }^{7,10}$ In sorghum, we find some structural characters of the ETC that fit it for substance transport. i) Only the side and bottom walls of the ETC near the maternal tissues form wall ingrowths. They are less and less from the outermost layer to the inner, and form firstly in the outermost layer and later in the inner layer. Thus, the ability for substance transportation becomes weaker and weaker. The solute concentration gradient between cells forms, which promotes nutrient transporta- tion. ii) There are many mitochondria and dense endoplasmic reticulum surrounding the wall ingrowths. Mitochondria probably provide energy for substance exchange. The endoplasmic reticulum probably divides the cytoplasm into parts of different concentrations. Therefore, a solute concentration gradient in the cells forms, which promotes nutrient transport further. iii) A part of the nucleus opposite the wall ingrowths invaginates and the endoplasmic reticulum near the nucleus distributes along the invaginating direction. The two results prove that the solute is being transported in the ETC.

In addition, ETC have a tight relationship with the embryo and endosperm during the development of sorghum caryopses. Therefore, ETC play an important part in nutrient transport during the development of sorghum caryopses.

\section{References}

1. Gunning BES, Pate JS. "Transfer cells": plant cells with wall ingrowths, specialized in relation to short distance transport of solutes - their occurrence, structure, and development. Protoplasma 1969;68:107-33.

2. Rost TL, Lersten NR. Transfer aleurone cells in Setaria lutenscens (Gramineae). Protoplasma 1970;71:403-8.

3. Zee SY, 0'Brien TP. Aleurone transfer cells and other structural features of the spikelet of millet. Aust J Biol Sci 1971;24:391-5.

4. Cochrane MP, Duffus CM. The nucellar projection and modified aleurone in the crease region of developing caryopses of barley (Hordeum vulgare L var disitichum). Protoplasma 1980;103:36175.

5. Doan DNP, Linnestad C, Olsen OA. Isolation of molecular markers from the barley endosperm coenocyte and the surrounding nucellus cell layers. Plant Mol Biol 1996;31:877-6.

6. Weschke W, Panitz R, Gubatz S, et al. The role of invertases and hexose transporters in controlling sugar ratios in maternal and filial tissues of barley caryopses during early development. Plant J 2003;33:395411.

7. Thompson RD, Hueros G, Becker H, et al. Development and functions of seed transfer cells. Plant Sci 2001;160:775-83.

8. Davis RW, Smith JD, Cobb BG. A light and electron microscope investigation of the transfer cell region of maize caryopses. Can J Bot 1990;68:471-9.

9. Becraft PW. Cell fate specification in the cereal endosperm. Semin Cell Dev Biol 2001;12:387-94.

10. Pate JS, Gunning BES. Transfer cells. Annu Rev Plant Physiol 1972;23:173-96.

11. Royo J, Gómez E, Hueros G. Transfer Cells. In: Plant Cell Monographs. Olsen 0-A, ed. Berlin Heidelberg: Springer-Verlag, 2007, monogr 8, 73-89.

12. Felker FC, Shannon JC. Movement of 14Clabeled assimilates into kernels of Zea mays L. III. An anatomical examination and microautoradiographic study of assimilate transfer. Plant Physiol 1980;65:864-70. 